This is an electronic reprint of the original article. This reprint may differ from the original in pagination and typographic detail.

\author{
Author(s): Uhlig, J.; Fullagar, W.; Ullom, J.N.; Doriese, W.B.; Fowler, J.W.; Swetz, D.S.; Gador, N.; \\ Canton, S.E.; Kinnunen, Kimmo; Maasilta, Ilari; Bennett, C.D.Reintsema D.A.; Vale, L.R.; \\ Hilton, G.C.; Irwin, K.D.; Schmidt, D.R.; Sundström, V.
}

Title: $\quad$ Table-top ultrafast X-ray microcalorimeter spectrometry for molecular structure

Year: $\quad 2013$

Version:

Please cite the original version:

Uhlig, J., Fullagar, W., Ullom, J.N., Doriese, W.B., Fowler, J.W., Swetz, D.S., Gador, N., Canton, S.E., Kinnunen, K., Maasilta, I., Bennett, C. D., Vale, L.R., Hilton, G.C., Irwin, K.D., Schmidt, D.R., \& Sundström, V. (2013). Table-top ultrafast X-ray microcalorimeter spectrometry for molecular structure. Physical Review Letters, 110, Article 138302. https://doi.org/10.1103/PhysRevLett.110.138302

All material supplied via JYX is protected by copyright and other intellectual property rights, and duplication or sale of all or part of any of the repository collections is not permitted, except that material may be duplicated by you for your research use or educational purposes in electronic or print form. You must obtain permission for any other use. Electronic or print copies may not be offered, whether for sale or otherwise to anyone who is not an authorised user. 


\title{
Table-Top Ultrafast X-Ray Microcalorimeter Spectrometry for Molecular Structure
}

\author{
J. Uhlig, ${ }^{1,2}$ W. Fullagar, ${ }^{1}$ J. N. Ullom, ${ }^{2}$ W. B. Doriese, ${ }^{2}$ J. W. Fowler, ${ }^{2}$ D. S. Swetz, ${ }^{2}$ N. Gador, ${ }^{1}$ S. E. Canton, ${ }^{1,3}$ \\ K. Kinnunen, ${ }^{1,4}$ I. J. Maasilta, ${ }^{4}$ C. D. Reintsema, ${ }^{2}$ D. A. Bennett, ${ }^{2}$ L. R. Vale, ${ }^{2}$ G. C. Hilton, ${ }^{2}$ \\ K. D. Irwin, ${ }^{2}$ D. R. Schmidt, ${ }^{2}$ and V. Sundström ${ }^{1}$ \\ ${ }^{1}$ Department of Chemical Physics, Lund University, Lund, Sweden \\ ${ }^{2}$ National Institute of Standards and Technology, 325 Broadway MS 817.03, Boulder, Colorado 80305, USA \\ ${ }^{3}$ Department of Synchrotron Radiation Instrumentation, Lund University, Lund, Sweden \\ ${ }^{4}$ Nanoscience Center, Department of Physics, P.O. Box 35, FI-40014 University of Jyväskylä, Jyväskylä, Finland
}

(Received 18 October 2012; published 26 March 2013)

\begin{abstract}
This work presents an x-ray absorption measurement by use of ionizing radiation generated by a femtosecond pulsed laser source. The spectrometer was a microcalorimetric array whose pixels are capable of accurately measuring energies of individual radiation quanta. An isotropic continuum X-ray spectrum in the few-keV range was generated from a laser plasma source with a water-jet target. $\mathrm{X}$ rays were transmitted through a ferrocene powder sample to the detector, whose pixels have average photon energy resolution $\Delta E=3.14 \mathrm{eV}$ full-width-at-half-maximum at $5.9 \mathrm{keV}$. The bond distance of ferrocene was retrieved from this first hard-x-ray absorption fine-structure spectrum collected with an energydispersive detector. This technique will be broadly enabling for time-resolved observations of structural dynamics in photoactive systems.
\end{abstract}

DOI: 10.1103/PhysRevLett.110.138302

PACS numbers: 82.80.Ej, 07.85.-m, 61.05.cj, 78.47.D-

Observing and understanding the motion of atoms and electrons on pico-, femto-, and attosecond time scales is a frontier of contemporary science and the motivation for vigorous development of ultrafast time-resolved spectroscopic techniques based on hard $\mathrm{x}$ rays [1]. These techniques are often flux-limited, particularly with laboratory pulsed laser x-ray sources. Long accumulation periods are required to achieve the necessary energy resolution owing to the involvement of Bragg reflection with its generally narrow-bandpass characteristics and consequent dramatic flux losses [2-6]. Direct energy-resolving detectors such as calorimeters or charge-coupled devices have a much higher quantum yield and a long history in radiation studies $[7,8]$. Recent technological developments in microcalorimetric detectors [9] have improved the energy resolution and count rates of these highly efficient direct photonmeasurement schemes sufficiently to enable useful measurements in reasonable time frames.

The novel combination of a pulsed-laser-generated, broadband, hard x-ray source with a photon-measuring cryogenic microcalorimeter array has been motivated previously [10-13]. It promises spectroscopic ultrafast structural measurements with photon-counting rates that, in the prototype version discussed in this Letter, are comparable to established techniques such as electron-beam slicing at synchrotrons and focusing-crystal geometries $[1,14]$. The strength of this new approach lies in its straightforward scalability combined with very high collection efficiency. X-ray absorption fine structure (XAFS) features are observable and quantifiable, as are extended range modulations that result from the influence of adjacent atoms on the absorption dipole strength, and contain molecular structure and valence orbital spectroscopic information. The setup allows simultaneous observation of multiple absorption edges and considerable flexibility for binning during and after the experiment. This demonstration is a critical development in the advancement of inhouse molecular structure analysis, particularly for subpicosecond pump-probe studies in condensed phases $[3,10-12,15]$. This publication aims to demonstrate the new opportunities and presents, for the first time, a hard x-ray absorption fine-structure spectrum recorded with a highly efficient energy-dispersive detector.

The $\mathrm{x}$-ray plasma source is based on isotropic continuum bremsstrahlung generated when focusing $1 \mathrm{kHz}, 800 \mathrm{~nm}$ Ti:sapphire laser pulses with $2.5 \mathrm{~mJ} /$ pulse and $\sim 50 \mathrm{fs}$ duration onto a flowing water jet. The jet is operated at water aspirator vacuum pressure $\left(\sim 25\right.$ torr at $\left.21^{\circ} \mathrm{C}\right)$ and enclosed by a target chamber constructed from $8 \mathrm{~mm}$ thick aluminum. The flux, spectral, and temporal properties of the $\mathrm{x}$ rays emitted at this laser pulse energy were described previously [10,11]. Efforts were made to ensure sufficient temporal contrast when operating the laser, to avoid generation of high-energy electron beams and concomitant reduction of target-generated x-ray flux [16]. The laser system provides up to $6 \mathrm{~mJ} /$ pulse and thus has sufficient additional energy to enable optical stimulation of samples in a pump-probe arrangement.

Calorimetric event detection is based on the thermalization of single photons in an absorber and the observation of the subsequent thermal flow. The particular detector array used here is based on bismuth absorbers with transition-edge-sensor (TES) thermometers operating at millikelvin temperatures [17]. Pixels are read out in a 
time-division-multiplexing scheme [18], asynchronously to the laser pulses, and with signal-record lengths of $\approx 12 \mathrm{~ms}$, during which pileup had to be rejected. The laser source can provide $\mathrm{x}$-ray events in each pixel at up to the laser repetition rate $(1 \mathrm{kHz})$, but the source-detector distance and intervening filters were adjusted to limit the count rate. The record length is a compromise between energy resolution and readout speed. It is not a fundamental parameter, but rather reflects the current state of the readout electronics, pixel recovery periods, and pulseprocessing approach. Each of these quantities is the subject of current development, and significant improvements are anticipated. The bismuth x-ray absorber in each pixel has dimensions of $350 \times 350 \times 2.5 \mu \mathrm{m}$ and stops $\approx 53 \%$ of the arriving photons at $7.2 \mathrm{keV}$. From the installed array with 160 pixels, 22 detectors contributed to the presented spectra. A system with all 160 pixels and shorter record lengths is currently under characterization and will provide more than 16 times shorter accumulation periods. Pixel centers are positioned on a square $665 \mu \mathrm{m}$ grid in a roughly circular array, with each absorber masked by a $320 \times 305 \mu \mathrm{m}$ aperture.

The emission lines from $\mathrm{Mn}-K \alpha$ at $5.9 \mathrm{keV}$ measured with the 22 pixels detector show an average energy resolution of $\Delta E_{\mathrm{FWHM}}=3.14 \mathrm{eV}$. During laser operation, the average resolution was $3.4 \mathrm{eV}$. The difference between these values is explained by a higher count rate from the laser as well as electromagnetic pickup from laser operation. The absorber and thermometer parameters are chosen to give the best energy resolution up to a maximum working energy of $\approx 10 \mathrm{keV}$, where the TES is thermally saturated by a single event. For a given device the energy resolution is roughly constant over the full working range of the device. The maximum working energy $E_{\max }$ is chosen during device design by adjusting film thicknesses and other TES parameters. The resolution $\Delta E$ of different designs scales as $\sim \sqrt{E_{\max }}[9,17]$. The detector array is cooled by a two-stage adiabatic-demagnetization refrigerator (ADR) that is in turn backed by a cryogen-free pulse-tube cryocooler. The ADR can provide $\mathrm{a} \approx 35 \mathrm{mK}$ base temperature but is typically operated at $85 \mathrm{mK}$. Each sensor, which has a critical temperature of $120 \mathrm{mK}$, is maintained in its resistive transition via Joule heating from an applied voltage bias. The average operation period before recycling the ADR is $14 \mathrm{~h}$ preceded by $\mathrm{a} \approx 1 \mathrm{~h}$ adjustment and calibration period. The recycling period is $2-3 \mathrm{~h}$.

For the very first spectrum, ferrocene $\mathrm{Fe}\left(\mathrm{C}_{5} \mathrm{H}_{5}\right)_{2}$ was chosen. Ferrocene was studied in the early days of $\mathrm{x}$-ray absorption spectroscopy $[19,20]$ and remains a subject of interest in studies of organometallic compounds [21] and solar-cell applications [22]. With its weakly scattering carbon atoms it can serve as a model complex for many metal-containing organic compounds.

Figure 1 shows the first hard $\mathrm{x}$-ray absorption finestructure spectrum collected by an energy-dispersive detector with sufficient resolution and statistics to retrieve molecular structure information. After digital filtering and rejection of pileup and other artifacts it contains $\sim 8.9 \times 10^{6}$ events accumulated at $\sim 175$ counts/s. Of these, $\sim 7.3 \times 10^{6}$ lie outside the energy range of interest

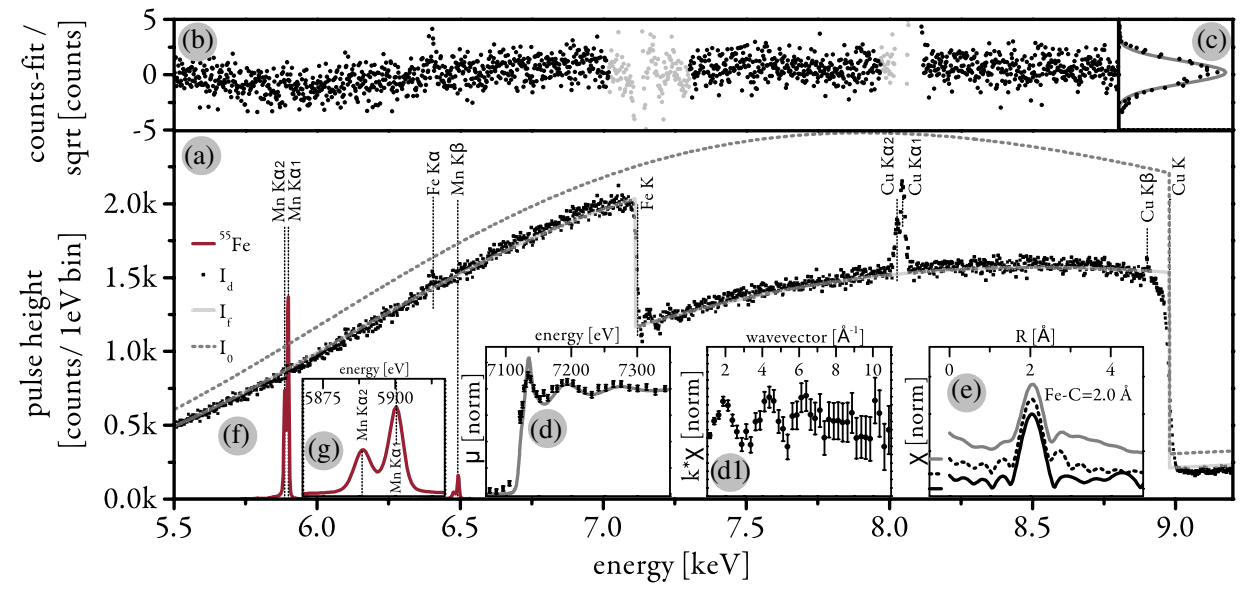

FIG. 1 (color online). (a) Laser plasma absorption spectrum of ferrocene in transmission mode. Included are emission lines and absorption edges of metal filters (see text). Dashed line $\left(I_{0}\right)$ is the fitted spectrum without the sample. (b) Difference between measured spectrum $\left(I_{\mathrm{d}}\right)$ and the reference fit $\left(I_{\mathrm{f}}\right)$ of all involved materials, normalized to the Poisson error. Areas of emission lines and fine structure modulation (gray) were excluded. (c) A histogram of the normalized difference, with a Gaussian $1 \sigma$ curve superimposed. (d) Absorption fine structure of ferrocene, normalized to a measured background spectrum and the edge jump with indicated Poissonlimited error bars. The gray line is a synchrotron reference measurement from the same compound. (d1) $k \chi$ presented in $k$ space to show the quality of the data. The error bars were generated using the statistical fluctuation expected for the number of samples per bin. (e) $|\chi|$ as function of $R$ from the data (black line), single path fit (dotted black line) and synchrotron comparison data (gray line). Curves are offset vertically and the zero levels are marked; for details see text. (f) Additional calibration lines measured prior to the absorption spectrum; the Mn- $K \alpha$ splitting is clearly resolvable as shown in $(\mathrm{g})$. 
for XAFS, showing the importance of suppressing unwanted X-ray energies. In this experiment some suppression was accomplished by a stack of aluminum and copper foils, but significant improvement is possible.

The combination of source spectrum, copper, and aluminium filters, and the sample material accounts for the overall features of the spectrum in Fig. 1 (see the Supplemental Material [23] for a sketch of the experiment). Multiple absorption edges are observable simultaneously in a given measurement, a valuable feature for future experiments. The spectral range could be extended down to $\approx 200 \mathrm{eV}$, which includes the whole class of sulphurous materials, the $K$ and $L$ edges of the $3 \mathrm{~d}$ transition metals, and the L edges of many heavier elements. The $200 \mathrm{eV}$ lower bound is determined mainly by transmission through three infrared filter windows and one vacuum window on the cryostat. The region of primary interest in this experiment was $7000-8000 \mathrm{eV}$. The obvious lines in the spectra arise from fluorescence from a copper foil (whose position was close to the detector). The Mn-K $\alpha$ lines from a ${ }^{55} \mathrm{Fe}$ source shown in Figs. 1(f) and 1(g) were recorded prior to the absorption spectrum, and are essential for the energy and temperature calibration of the detector. Each pixel is calibrated separately, and its energy resolution can be retrieved from the known fine structure of the emission lines [24]. This calibration has to be done either on features of the measured spectrum or during a separate short calibration period. Sparse calibration features can introduce small systematic errors in the detected energies.

Fine-structure oscillations above the $\mathrm{Fe} K \alpha$ (ferrocene) and $\mathrm{Cu} K \alpha$ (filter) edges are visible in the data and show that two widely separated edges can be observed simultaneously. Figure 1(a) also shows a fit (gray solid line) to the baseline (using an estimate of the absorption of a free Fe atom). Residuals divided by their Poisson error are shown in Fig. 1(b), areas omitted from the noise histogram shown in Fig. 1(c) are marked in gray. The excellent agreement between the histogram and a superimposed Gaussian curve with $\sigma=1$, shown in Fig. 1(c), demonstrates that Poisson noise associated with the number of photons per bin is the dominant noise source. Figure 1(d) shows the absorption fine structure of ferrocene, after normalization to a spectrum without the sample and the edge jump. The error bars for each bin (equidistant in $k$ space) were obtained from the Poisson error of the recorded number of photons. The gray line in this panel shows the excellent agreement with the synchrotron reference measurement taken from the same compound. The data converted into $k$ space are presented in Fig. 1(d1). Again the error bars are the Poisson error of the number of photons per bin. This spectrum shows the influence of the limited statistics in this very first spectrum. In Fig. 1(e) the data have been converted into $R$ space with a window from $k=1-8 \AA^{-1}$, which is suitable for first-shell analysis and corrected for the $\mathrm{Fe}-\mathrm{C}$ phase shift (solid black line).
Calculation of the single scattering path from the iron to the nearest carbons in ferrocene by use of FEFF6 [25] and optimization in $k$ space by use of single $k$ weight with the fitting routine in ARTEMIS [26] of the IFEFFIT [27] package yielded the fit shown by the black dotted line; synchrotron data from the same compound are shown for comparison (gray line). The latter two graphs are offset vertically and the zero values are marked. All show agreement with the known Fe-C bond distance of $2.0 \AA[19,28]$.

The extended $\mathrm{x}$-ray-absorption fine structure spectroscopy (EXAFS) function $\chi(E)=\left[\mu(E)-\mu_{0}(E)\right] / \mu_{0}(E)$ and its error follow from the absorption coefficients $\mu(E)=\ln \left[I_{0}(E) / I_{\mathrm{d}}(E)\right]$ and $\mu_{0}(E)=\ln \left[I_{0}(E) / I_{\mathrm{f}}(E)\right]$, where $I_{\mathrm{d}}=$ data $=$ integer, transmitted number of counts (experimental noise $=\sqrt{I_{\mathrm{d}}}$ ), $I_{\mathrm{f}}=$ fitted free-atom spectrum, including sample contribution (no experimental noise), and $I_{0}=$ fitted free-atom spectrum, excluding sample contribution (no experimental noise).

These give

$$
\chi(E)=\frac{\ln \left(I_{\mathrm{f}} / I_{\mathrm{d}}\right)}{\ln \left(I_{0} / I_{\mathrm{f}}\right)}, \quad \text { with error: } \sigma(\chi)=\frac{1}{\ln \left(I_{0} / I_{\mathrm{f}}\right)} \frac{1}{\sqrt{I_{\mathrm{d}}}} ;
$$

$\chi(E)$ is a ratio of intensities and an intrinsic property of the sample that distinguishes the true sample from its freeatom approximation. If it has amplitude $A$ evaluated at the energy where a feature of interest is present, the signal-tonoise ratio (SNR) for measurements is then

$$
\mathrm{SNR}=\frac{A}{\sigma(\chi)}=A \sqrt{I_{\mathrm{d}}} \ln \left(I_{0} / I_{\mathrm{f}}\right)
$$

By choosing the sample thickness and concentration such that $I_{\mathrm{d}}\left(\approx I_{\mathrm{f}}\right) \approx I_{0} / e^{2}$, and thus $\ln \left(I_{0} / I_{\mathrm{f}}\right) \approx 2$, an optimum signal is obtained [29]. Incorporating this, and expanding $I_{\mathrm{d}}$ gives

$$
\mathrm{SNR}=2 A \sqrt{t_{\text {meas }} N_{\text {pix }} \rho S(B)},
$$

where $t_{\text {meas }}$ is the measurement period, $N_{\text {pix }}$ is the number of pixels, and $\rho$ is the average pixel event rate over the entire measured spectrum. The function $S(B)=I_{\mathrm{d}, \mathrm{B}} / I_{\mathrm{d}, \text { tot }}$ is the relative spectral density of the measured spectrum, where we have a recording whose total integrated intensity is $I_{\mathrm{d}, \text { tot }}$, and whose intensity is $I_{\mathrm{d}, \mathrm{B}}$ in a particular bin of energy width $B$, chosen to contain the feature of interest. $S(B)$ shows the advantages of selective binning and the importance of filtering out unwanted energies from the measurement. As EXAFS chemical interpretations are done in the photoelectron momentum $(k)$ space, $k \sim \sqrt{\left(E-E_{\text {edge }}\right)}$, the choice of linear binning in $k$ space improves the SNR at higher $k$ values compared to linear binning in $E$. This can be easily optimized during data analysis, since the data are not prebinned by the dataacquisition method.

For steady-state measurements, amplitude $A$ is the change in absorption between the actual sample and a 
free-atom description of the sample, normalized by the depth of the edge jump. For pump-probe x-ray-absorption spectroscopy (XAS) measurements, which are the motivation of the present lab-based developments, the signal scales with the fraction of molecules that were excited into the desired state. In selected systems, the signal can be in the range of several percent normalized to the edge jump; for the strongest feature during the low spin-high spin conversion of $\mathrm{Fe}(\mathrm{bpy})_{3}$ a change of $\sim 2.6 \%$ in a $25 \mathrm{mM}$ liquid jet under otherwise similar experimental condition was observed [1]. The ferrocene measurement presented in this work should be comparable: here we have $t=\sim 5 \times 10^{4} \mathrm{~s}, N_{\text {pix }}=22$ pixels, $\bar{r}=\sim 8 / \mathrm{s}, I_{\mathrm{d}, \mathrm{B}}=$ $\sim 1500$ events $/ 1 \mathrm{eV}$ bin, and $I_{\text {tot }}=\sim 8.94 \times 10^{6}$ events, suggesting $\mathrm{SNR} \approx 0.9$. For the $5 \mathrm{eV}$ bin as at $7150 \mathrm{eV}$ in Fig. 1(d) (binned to equal $k$ steps), the SNR improves to 2. With the next upgrades, the measurement period for data such as in Fig. 1 will be reduced to below $30 \mathrm{~min}$. For the foreseeable future, the signal-to-noise ratio of the XAFS signal will be limited by the number of pixels, because the peak count rate (if indifferent to pileup) is limited to 1 photon/pixel/laser pulse. Future iterations will increase the fill factor and efficiency of the detector to allow experiments with even lower flux or stronger filtering. $\mathrm{X}$-ray optics such as polycapillary or other broadband lenses [30] might increase the captured solid angle and decouple the sample from the generation environment with slight loss of temporal resolution [31]. Temporal smearing is not an issue if the optic is placed between sample and detector but has to be considered if placed between source and sample.

The approach described here is compatible with a broad range of samples and sample phases. When combined with an optical pump in a pump-probe arrangement as being developed, it can observe fundamental ultrafast chemistry at atomic resolution. For these reasons, and because of its compatibility with a conventional laboratory setting, the technique is well suited for dissemination. All detector parameters have been dramatically surpassed in recent developments. For example, detectors with energy resolution as good as $1.6 \mathrm{eV}$ at $5.9 \mathrm{keV}$ have now been demonstrated [32]. Improved multiplexing techniques such as code-division multiplexing (CDM) and the integration of $\mathrm{CDM}$ with microwave resonant techniques promise continued increases in array scale into the megapixel regime $[33,34]$. Such arrays will reduce integration periods by $10^{5}$ compared to the results shown here.

To conclude, a cryogenic microcalorimeter array has been coupled to a laser-generated radiation source. A hard $\mathrm{x}$-ray absorption fine-structure spectrum with sufficient energy resolution to extract intermolecular bond lengths has been collected for the first time with an energydispersive technique. The observed EXAFS oscillation and the retrieved bond length are consistent with synchrotron data within error bars $[19,28]$. The detectors do not suffer from narrow-band acceptance losses, do not require careful mechanical alignment, and are in a state of rapid development. These facts distinguish them from conventional detection topologies based on Bragg reflection. An excellent free-atom fit to the data was obtained, from which the incident spectrum and absence of excess noise sources were confidently established. The source spectrum is well described by a simple functional form over a multi$\mathrm{keV}$ energy range, thus establishing the suitability of the source for broadband-absorption spectroscopies. A general expression for the signal-to-noise ratio in measurements of this type was derived and applied to the data. The prospects for lab-based molecular structure studies are excellent and imminent using this and related approaches, particularly for ultrafast studies [12], and are advancing in step with widespread implementations of laser-driven sources and contemporary developments of cryogenic microcalorimeter arrays.

The system was developed in the collaborative environment of the Lund Laser Centre and the Quantum Device Group of NIST Boulder, and acknowledges funding from the Swedish Consortium for Artificial Photosynthesis, the NIST Innovations in Measurement Science program and an ERC Advanced Investigator Grant No. 226136 to V. S. J U. acknowledges a scholarship under the Marie Curie Early Stage Researcher Training Site MAXLAS that was financed by the European Commission under the 6th European Framework Program.

[1] C. Bressler and M. Chergui, Chem. Rev. 104, 1781 (2004).

[2] F. Ráksi, K. R. Wilson, Z. Jiang, A. Ikhlef, C. Y. Côté, and J.-C. Kieffer, J. Chem. Phys. 104, 6066 (1996).

[3] T. Lee, Y. Jiang, C. G. Rose-Petruck, and F. Benesch, J. Chem. Phys. 122, 084506 (2005).

[4] L. v. Hamos, Naturwissenschaften 20, 705 (1932).

[5] T. Missalla, I. Uschmann, E. Förster, G. Jenke, and D. von der Linde, Rev. Sci. Instrum. 70, 1288 (1999).

[6] V.A. Arkadiev, A.A. Bjeoumikhov, M. Haschke, N. Langhoff, H. Legall, H. Stiel, and R. Wedell, Spectrochim. Acta B Atom. Spectros. 62, 577 (2007).

[7] J. Tyndall, Heat: A Mode of Motion (D. Appleton, New York, 1895).

[8] R. Balian and J.-C. Adam, Proceedings of the Les Houches Summer School (North-Holland, Amsterdam, 1982).

[9] J. N. Ullom, J. A. Beall, W. B. Doriese, W. D. Duncan, L. Ferreira, G. C. Hilton, K. D. Irwin, C. D. Reintsema, and L. R. Vale, Appl. Phys. Lett. 87, 194103 (2005).

[10] W. K. Fullagar, M. Harbst, S. Canton, J. Uhlig, M. Walczak, C.-G. Wahlström, and V. Sundström, Rev. Sci. Instrum. 78, 115105 (2007).

[11] W. Fullagar, J. Uhlig, M. Walczak, S. Canton, and V. Sundström, Rev. Sci. Instrum. 79, 103302 (2008).

[12] W. Fullagar, J. Uhlig, N. Gador, K. Kinnuen, I. Maasilta, C.-G. Wahlström, and V. Sundstrom, AIP Conf. Proc. 1234, 919 (2010). 
[13] W. K. Fullagar, D.M. Paganin, and C.J. Hall, arXiv:1102.4680.

[14] F. Benesch, T. Lee, Y. Jiang, and C. G. Rose-Petruck, Opt. Lett. 29, 1028 (2004).

[15] I. V. Tomov, D. A. Oulianov, P. Chen, and P. M. Rentzepis, J. Phys. Chem. B 103, 7081 (1999).

[16] J. Uhlig, C.-G. Wahlström, M. Walczak, V. Sundström, and W. Fullagar, Laser Part. Beams 29, 415 (2011).

[17] K. D. Irwin and G. C. Hilton, Cryogenic Particle Detection, edited by C. Enss (Springer-Verlag, New York, 2005) p. 81.

[18] P. A. J. de Korte, J. Beyer, S. Deiker, G. C. Hilton, K. D. Irwin, M. MacIntosh, S. W. Nam, C. D. Reintsema, L. R. Vale, and M. E. Huber, Rev. Sci. Instrum. 74, 3807 (2003).

[19] S. P. Cramer, T. K. Eccles, F. Kutzler, K. O. Hodgson, and S. Doniach, J. Am. Chem. Soc. 98, 8059 (1976).

[20] M. F. Ruiz-Lopez, M. Loos, J. Goulon, M. Benfatto, and C. R. Natoli, Chem. Phys. 121, 419 (1988).

[21] M. Balasubramanian, M. T. Giacomini, H. S. Lee, J. McBreen, and J.H. Sukamto, J. Electrochem. Soc. 149, D137 (2002).

[22] T. Daeneke, T.-H. Kwon, A. B. Holmes, N. W. Duffy, U. Bach, and L. Spiccia, Nat. Chem. 3, 211 (2011).

[23] See Supplemental Material at http://link.aps.org/ supplemental/10.1103/PhysRevLett.110.138302 for a detailed description of the experimental setup.
[24] G. Hölzer, M. Fritsch, M. Deutsch, J. Härtwig, and E. Förster, Phys. Rev. A 56, 4554 (1997).

[25] S. Zabinsky, J. Rehr, A. Ankudinov, R. Albers, and M. Eller, Phys. Rev. B 52, 2995 (1995).

[26] B. Ravel and M. Newville, J. Synchrotron Radiat. 12, 537 (2005).

[27] M. Newville, J. Synchrotron Radiat. 8, 322 (2001).

[28] P. Seiler and J. D. Dunitz, Acta Crystallogr. Sect. B 35, 1068 (1979).

[29] R. G. Shulman, P. Eisenberger, and B. M. Kincaid, Annu. Rev. Biophys. Biophys. Chem. 7, 559 (1978).

[30] M. Kumakhov, Phys. Rep. 191, 289 (1990).

[31] M. Bargheer, N. Zhavoronkov, R. Bruch, H. Legall, H. Stiel, M. Woerner, and T. Elsaesser, Appl. Phys. B 80, 715 (2005).

[32] S. R. Bandler et al., in Proceedings of SPIE 7732, Space Telescopes and Instrumentation 2010: Ultraviolet to Gamma Ray (SPIE, San Diego, 2010), p. 38.

[33] K. D. Irwin, H. M. Cho, W. B. Doriese, J. W. Fowler, G. C. Hilton, M. D. Niemack, C. D. Reintsema, D. R. Schmidt, J. N. Ullom, and L. R. Vale, J. Low Temp. Phys. 167, 588 (2012).

[34] G. M. Stiehl, W. B. Doriese, J. W. Fowler, G. C. Hilton, K. D. Irwin, C. D. Reintsema, D. R. Schmidt, D. S. Swetz, J. N. Ullom, and L. R. Vale, Appl. Phys. Lett. 100, 072601 (2012). 